Bull. Korean Math. Soc. 44 (2007), No. 4, pp. 871-878

\title{
PRESENTING MATRICES OF MAXIMAL COHEN-MACAULAY MODULES
}

\author{
Kisuk LeE
}

\begin{abstract}
We define a numerical invariant row $C M(A)$ over Cohen-Macaulay local ring $A$, which is related to rows of the presenting matrices of maximal Cohen-Macaulay modules without free summands. We show that $\operatorname{row}(A)=\operatorname{row}_{C M}(A)$ for a Cohen-Macaulay (not necessarily Gorenstein) local ring $A$.
\end{abstract}

\section{Introduction}

Throughout this paper, we assume that $(A, \mathfrak{m})$ is a Noetherian local ring, and all modules are unitary.

It is proved in [3] that there are certain restrictions on the entries of the maps in the minimal free resolutions of finitely generated modules of infinite projective dimension over Noetherian local rings. This fact provides not only a new way to understand some previously known results in commutative ring theory (see for instance [3, Corollary 2.8], or [3, Proposition 2.2]), but also new interesting invariants of local rings. These invariants have turned out to be quite useful; for example, the Auslander index of $A$ can be described as a column invariant when $A$ is Gorenstein ([5]), and the multiplicity of $A$ can be also explained by these invariants when $A$ is hypersurface. (For the further background of these invariants, we refer the reader to $[3,4]$ and [6].)

In [1], he shows that a Cohen-Macaulay ring of finite index, with a canonical module, is Gorenstein on the punctured spectrum. Leuschke ([8]) generalizes Ding's result: the result remains true under the weaker hypothesis that a ring has a Gorenstein module. In [5], it is shown that index $(A)$ is the same as $\operatorname{col}_{C M}(A)$, and so a Cohen-Macaulay ring with Gorenstein module such that $\operatorname{col}_{C M}(A)$ is finite must be Gorenstein on the punctured spectrum. This paper particularly deals with invariants $\operatorname{row}(A), \operatorname{row}_{C M}(A)$, and we will show that a Cohen-Macaulay ring such that $\operatorname{row}_{C M}(A)$ is finite is not necessarily Gorenstein on the punctured spectrum.

Received May 15, 2007.

2000 Mathematics Subject Classification. 13H10, 13C14, 13D02.

Key words and phrases. column and row invariants, maximal Cohen-Macaulay modules, syzygy modules, Cohen-Macaulay ring.

The author was supported by 2006 Sookmyung Women's University Research Grant. 
We recall that $\operatorname{row}(A)$ is defined with the rows of the maps in infinite minimal resolutions: $\operatorname{row}(A)$ is the smallest integer $t \geq 1$ such that for each finitely generated $A$-module $M$ of infinite projective dimension, each row of $\varphi_{i}$ contains an element outside $\mathrm{m}^{t}$ for all $i>\operatorname{depth} A$, where $\varphi_{i}$ is the $i$-th map of a minimal free resolution of $M$. (When $A$ is regular, we let $\operatorname{row}(A)=1$. It is shown ([4]) that $\operatorname{row}(A)$ is the same as $\operatorname{col}(A)$ if $A$ is Gorenstein.) In [7], it was shown that $\operatorname{col}(A)$ can be described in terms of the columns of presenting matrices of maximal Cohen-Macaulay modules without free summands when $A$ is Gorenstein. In this article, we show that row $(A)$ can be also described in terms of the rows of presenting matrices of maximal Cohen-Macaulay modules (not necessarily without free summand) when $A$ is Cohen-Macaulay (not necessarily Gorenstein).

In Section 1, we give the definitions of $\operatorname{row}_{j}(A)$ and $\operatorname{row}_{C M}(A)$ in detail, and study some basic properties of these invariants. In particular, we investigate the behavior of these invariants under flat base change.

In Section 2, we prove that $\operatorname{row}(A)=\operatorname{row}_{C M}(A)$ for a Cohen-Macaulay local $\operatorname{ring} A$ (we note that $\operatorname{col}(A)=\operatorname{col}_{C M}(A)$ was shown when $A$ is Gorenstein ([7])), which explains row $(A)$ can be described in terms of the rows of presenting matrices of maximal Cohen-Macaulay modules without free summands. We remark here that this is a somewhat interesting result comparing to a column invariant $\operatorname{col}(A)$ because $\operatorname{row}_{C M}(A)$ is always finite for a Cohen-Macaulay local ring $A$, while $\operatorname{col}_{C M}(A)$ is finite only when $A$ is Gorenstein on the punctured spectrum as we remark above.

To get this main result, we first show that $\operatorname{row}_{C M}(A)$ is the same as the supremum of $\operatorname{row}_{C M}(M)$ for maximal Cohen-Macaulay modules $M$ (not necessarily without free summands). The above fact gives us two corollaries that $\operatorname{row}_{C M}(A) \leq \operatorname{row}_{C M}(A / x A)$, where $x$ is a non zero-divisor of $A$, and $\operatorname{row}_{C M}(A)=\operatorname{row}_{C M}(A \llbracket X \rrbracket)$. The proof of the main result can be completed by using the mapping cone of complexes to carry information on matrices (depth $M$ )-steps to the left.

\section{Basic definitions and facts}

Let $M$ be a finitely generated $A$-module and $\varphi_{i}$ denote an $i$-th map of a minimal free resolution of $M: \cdots \longrightarrow A^{n_{2}} \stackrel{\varphi_{2}}{\longrightarrow} A^{n_{1}} \stackrel{\varphi_{1}}{\longrightarrow} A^{n_{0}} \longrightarrow M \longrightarrow 0$. A map $\varphi_{i}$ is represented by a matrix of size $n_{i+1} \times n_{i}$. By a (minimal) presenting matrix of $M$, we mean the representation matrix of $\varphi_{1}$. Then we note that $\varphi_{i}$ is represented by the presenting matrix of the $(i-1)$-st syzygy module.

Definition 1.1. Let $(A, \mathfrak{m})$ be a Cohen-Macaulay (non-regular) local ring, and $M$ a (nonfree) maximal Cohen-Macaulay module. We define $\operatorname{row}_{C M}(M)$ to be the smallest positive integer $c$ such that each row of the presenting matrix of $M$ contains an element outside $\mathfrak{m}^{c}$. We now define $\operatorname{row}_{C M}(A):=\sup \left\{\operatorname{row}_{C M}(M): M\right.$ is a maximal Cohen-Macaulay module without free summands\}. 
Definition 1.2. Let $(A, \mathfrak{m})$ be a Cohen-Macaulay local ring. For a nonnegative integer $j$

$\operatorname{row}_{j}(A):=\inf \{t \geq 1$ : for each $j$-th syzygy module without free summands of infinite projective dimension, each row of its presenting matrix contains an element outside $\left.\mathfrak{m}^{t}\right\}$.

If $A$ is regular, then we define $\operatorname{row}_{C M}(A)=1$, and $\operatorname{row}_{j}(A)=1$ for each nonnegative integer $j$. It is immediate from definitions that $\operatorname{row}(A)$, and $\operatorname{row}_{j}(A)$ are related as follows: $\operatorname{row}_{j}(A) \leq \operatorname{row}(A)<\infty$ for all $j \geq d$ and $\operatorname{row}_{j+1}(A) \leq$ $\operatorname{row}_{j}(A)$ for all $j \geq 0$.

We investigate the behavior of these invariants under flat base change. We first recall the following lemma, which deals with the behavior of maximal Cohen-Macaulay modules under flat base change:

Lemma $1.3([7])$. Let $f:(A, \mathfrak{m}) \rightarrow(B, \mathfrak{n})$ be a flat local homomorphism of Cohen-Macaulay local rings.

(1) If $M$ is a maximal Cohen-Macaulay A-module, then $M \otimes_{A} B$ is also a maximal Cohen-Macaulay $B$-module.

(2) If $M$ has no A-free summand, then $M \otimes_{A} B$ also has no $B$-free summand.

We establish inequalities between corresponding invariants under flat base change. The proof can be completed by the same way as the proof of Proposition 1.6 in [7].

Proposition 1.4. Let $f:(A, \mathfrak{m}) \rightarrow(B, \mathfrak{n})$ be a flat local homomorphism of Cohen-Macaulay local rings. Then

(1) $\operatorname{row}_{C M}(A) \leq \operatorname{row}_{C M}(B)$,

(2) $\operatorname{row}_{j}(A) \leq \operatorname{row}_{j}(B)$.

We note that the inequalities in the above proposition may be strict: Let $A=k \llbracket X \rrbracket /(X)$ and $B=k \llbracket X, Y \rrbracket /\left(X, Y^{2}\right)$, where $k$ is a field. Then $B$ is $A$ flat, and $\operatorname{row}_{0}(A)=\operatorname{row}_{C M}(A)=\operatorname{row}(A)=1<2=\operatorname{row}(B)=\operatorname{row}_{C M}(B)=$ row $_{0}(B)$ by Theorem 2.5 in this paper (see Section 2), and Example 2.12 in [4].

\section{Presenting matrix of maximal Cohen-Macaulay module}

The goal of this section is to show that $\operatorname{row}(A)=\operatorname{row}_{C M}(A)$ when $A$ is a Cohen-Macaulay local ring, i.e., row $(A)$ can be described in terms of the rows of presenting matrix of maximal Cohen-Macaulay modules without free summands. We remark here that $\operatorname{row}_{C M}(A)$ is always finite while $\operatorname{col}_{C M}(A)$ may be infinite. To get our main result we first show that $\operatorname{row}_{C M}(A)$ is the supremum of $\operatorname{row}_{C M}(M)$ for maximal Cohen-Macaulay modules $M$ (not necessarily without free summands).

The proof of the following proposition is quite elementary. However, we include a proof to see the behavior of entries of matrices after a change of bases. 
Proposition 2.1. Let $F_{1} \stackrel{\varphi_{1}}{\longrightarrow} F_{2} \stackrel{\varphi_{2}}{\longrightarrow} F_{3}$ be an exact sequence of free modules and homomorphisms. Then $\operatorname{Im}\left(\varphi_{2}\right)$ has a free summand if and only if $\varphi_{1}$ has a column of zeros after a suitable change of bases of $F_{2}$. In this case, every entry of some column of $\varphi_{1}$ can be replaced by all zeros after a suitable change of bases, and the other columns are remained unchanged.

Proof. Let $\left[a_{i j}\right]\left(\left[b_{i j}\right]\right)$ be an $\ell \times m(m \times n$, resp.) matrix which represents $\varphi_{1}\left(\varphi_{2}\right.$, resp.). Suppose that $\operatorname{Im}\left(\varphi_{2}\right)$ has a free summand, i.e., there is an onto homomorphism $\epsilon: \operatorname{Im}\left(\varphi_{2}\right) \rightarrow A$. We first note that $\operatorname{Im}\left(\varphi_{2}\right)$ is generated by $\eta_{1}, \ldots, \eta_{m}$ where $\eta_{i}=\left(b_{i 1}, \ldots, b_{i n}\right)$ is the $i$-th row of $\left[b_{i j}\right]$ for $i=1, \ldots, m$. Since $\epsilon$ is onto, there is some $i_{0}$ such that $\epsilon\left(\eta_{i_{0}}\right)$ is a unit, and so we may assume that $\epsilon\left(\eta_{i_{0}}\right)=1$. We show that every entry of the $i_{0}$-th column of $\varphi_{1}$ is zero after a suitable change of bases of $F_{1}$. We assume that $i_{0}=1$, i.e., $\epsilon\left(\eta_{1}\right)=1$. Now let $\eta_{i}^{*}=\eta_{i}-\epsilon\left(\eta_{i}\right) \cdot \eta_{1}$ for $i=2, \ldots, m$. Then for each $i=2, \ldots, m$

$$
\epsilon\left(\eta_{i}^{*}\right)=\epsilon\left(\eta_{i}-\epsilon\left(\eta_{i}\right) \cdot \eta_{1}\right)=\epsilon\left(\eta_{i}\right)-\epsilon\left(\eta_{i}\right) \epsilon\left(\eta_{1}\right)=0 .
$$

It is easy to show that $\operatorname{Im}\left(\varphi_{2}\right)$ is generated by $\eta_{1}, \eta_{2}^{*}, \ldots, \eta_{m}^{*}$. Let $\left[b_{i j}^{*}\right]$ be a matrix obtained from $\left[b_{i j}\right]$ by replacing its $i$-th row $\eta_{i}$ by $\eta_{i}^{*}$. In fact, $\left[b_{i j}^{*}\right]$ $=\left[e_{i j}\right]\left[b_{i j}\right]$, and a change of bases for $F_{2}$ with $\left[e_{i j}\right]$ transforms $\left[a_{i j}\right]$ to $\left[a_{i j}^{*}\right]=$ $\left[a_{i j}\right]\left[e_{i j}\right]^{-1}$ where

$$
\begin{gathered}
{\left[e_{i j}\right]_{m \times m}=\left[\begin{array}{cccccc}
1 & 0 & 0 & 0 & \cdots & 0 \\
-\epsilon\left(\eta_{2}\right) & 1 & 0 & 0 & \cdots & 0 \\
-\epsilon\left(\eta_{3}\right) & 0 & 1 & 0 & \cdots & 0 \\
\vdots & & & & & \vdots \\
-\epsilon\left(\eta_{m}\right) & 0 & 0 & 0 & \cdots & 1
\end{array}\right],} \\
{\left[e_{i j}\right]_{m \times m}^{-1}=\left[\begin{array}{cccccc}
1 & 0 & 0 & 0 & \cdots & 0 \\
\epsilon\left(\eta_{2}\right) & 1 & 0 & 0 & \cdots & 0 \\
\epsilon\left(\eta_{3}\right) & 0 & 1 & 0 & \cdots & 0 \\
\vdots & & & & & \vdots \\
\epsilon\left(\eta_{m}\right) & 0 & 0 & 0 & \cdots & 1
\end{array}\right]}
\end{gathered}
$$

Note that a given exact sequence is isomorphic to an exact sequence $F_{1} \stackrel{\varphi_{1}^{*}}{\longrightarrow}$ $F_{2} \stackrel{\varphi_{2}^{*}}{\longrightarrow} F_{3}$, where $\varphi_{1}^{*}\left(\varphi_{2}^{*}\right.$, resp.) is represented by $\left[a_{i j}^{*}\right]\left(\left[b_{i j}^{*}\right]\right.$, resp.).

Now it suffices to show that every entry of the first column of $\left[a_{i j}^{*}\right]$ is zero. We know that $\varphi_{2}^{*}\left(\varphi_{1}^{*}(1,0, \ldots, 0)\right)=(0, \ldots, 0)$ since $\varphi_{2}^{*} \circ \varphi_{1}^{*}=0$. Thus

$$
(0, \ldots, 0)=\varphi_{2}^{*}\left(\varphi_{1}^{*}(1,0, \ldots, 0)\right)=a_{11}^{*} \eta_{1}+a_{12}^{*} \eta_{2}^{*}+\cdots+a_{1 m}^{*} \eta_{m}^{*},
$$

and thus

$$
0=\epsilon\left(a_{11}^{*} \eta_{1}+a_{12}^{*} \eta_{2}^{*}+\cdots+a_{1 m}^{*} \eta_{m}^{*}\right)=a_{11}^{*} \cdot \epsilon\left(\eta_{1}\right)=a_{11}^{*} \cdot 1=a_{11}^{*} .
$$

Similarly we can show that $a_{1 j}^{*}=0$ for each $j=2, \ldots, \ell$, which implies that every entry of the first column of $\left[a_{i j}^{*}\right]$ is zero. By simple computations, we may see that the other columns are remained unchanged. 
Conversely, we assume that every entry of the $i$-th column of $\left[a_{i j}\right]$ is zero. When $\operatorname{Im}\left(\varphi_{2}\right)$ is generated by $\eta_{1}, \ldots, \eta_{m}$, we define a map

$$
\delta: \operatorname{Im}\left(\varphi_{2}\right) \rightarrow A \text { by } \delta\left(\eta_{k}\right)= \begin{cases}1, & k=i \\ 0, & k \neq i\end{cases}
$$

and $\delta\left(a_{1} \eta_{1}+\cdots a_{m} \eta_{m}\right)=a_{1} \delta\left(\eta_{1}\right)+\cdots+a_{m} \delta\left(\eta_{m}\right)$ for $a_{i} \in A$. We need to show that $\delta$ is well-defined to be a module homomorphism, i.e., if $a_{1} \eta_{1}+$ $\cdots+a_{m} \eta_{m}=(0, \ldots, 0)$, then $\delta\left(a_{1} \eta_{1}+\cdots+a_{m} \eta_{m}\right)=0$. Indeed, if $a_{1} \eta_{1}+$ $\cdots+a_{m} \eta_{m}=(0, \ldots, 0)$, then $\left(a_{1}, \ldots, a_{m}\right) \in \operatorname{Ker}\left(\varphi_{2}\right)=\operatorname{Im}\left(\varphi_{1}\right)=\operatorname{Im}\left(\left[a_{i j}\right]\right)$. Since every entry of the $i$-th column is zero, we must have $a_{i}=0$. Hence $\delta\left(a_{1} \eta_{1}+\cdots+a_{m} \eta_{m}\right)=a_{i}=0$. Since $\delta: \operatorname{Im}\left(\varphi_{2}\right) \rightarrow A$ is onto, $\operatorname{Im}\left(\varphi_{2}\right)$ has a free summand.

Now, we have the following result: $\operatorname{row}_{C M}(A)$ can be defined using maximal Cohen-Macaulay modules not necessarily without free summands.

Theorem 2.2. Let $(A, \mathfrak{m})$ be a Cohen-Macaulay local ring of dimension d. Then

$$
\operatorname{row}_{C M}(A)=\operatorname{row}_{C M}^{*}(A),
$$

where $\operatorname{row}_{C M}^{*}(A)=\sup \left\{\operatorname{row}_{C M}(M): M\right.$ is a maximal Cohen-Macaulay module $M\}$.

Proof. It is easy to see $\operatorname{row}_{C M}(A) \leq \operatorname{row}_{C M}^{*}(A)$ by the definition of $\operatorname{row}_{C M}(A)$. Now suppose $\operatorname{row}_{C M}^{*}(A)=t<\infty$. Then there is a maximal Cohen-Macaulay module $M$ such that every entry of some row of the presenting matrix of $M$ is contained in $\mathrm{m}^{t-1}$. If $M$ has no free summands, then we have $\operatorname{row}_{C M}(A) \geq t$. Suppose that $M=N \oplus A$. We claim that the presenting matrix of $N$ is obtained from the presenting matrix of $M$ by deleting some column. Suppose that $M$ is generated by $x_{1}, \ldots, x_{m}$, and $\epsilon: M \rightarrow A$ is an onto homomorphism such that $\epsilon\left(x_{i}\right)=1$ for some $i$. Then we can show that $N$ is generated by $m-1$ elements, and in fact $N \cong\left(x_{1}^{\prime}, \ldots, x_{i-1}^{\prime}, x_{i+1}^{\prime}, \ldots, x_{m}^{\prime}\right)$, where $x_{k}^{\prime}=x_{k}-\epsilon\left(x_{k}\right) x_{i}$ for $k=1, \ldots, i-1, i+1, \ldots, m$. By Proposition 2.1, every entry of the $i$-th column of the presenting matrix of $M$ is a zero. Then the submatrix $A^{*}$, which is obtained after deleting the $i$-th column of the presenting matrix of $M$, is the presenting matrix of $N$ since $\mu(N)=m-1$ and every entry of $A^{*}$ is in $\mathfrak{m}$. We may assume that $N$ is a maximal Cohen-Macaulay module without free summands (if not, we can do the same procedure). Since $A^{*}$ is a submatrix of the presenting matrix of $M$, every entry of some row of $A^{*}$ is contained in $\mathrm{m}^{t-1}$, and hence $\operatorname{row}_{C M}(A) \geq t=\operatorname{row}_{C M}^{*}(A)$.

Using the same argument as above, we are able to show that if one of them is infinite, then the other is also infinite.

In [4], $\operatorname{row}(A) \leq \operatorname{row}(A / x A)$ was proved for a non zero-divisor $x$ of $A$. The same property is also expected for $\operatorname{row}_{C M}(-)$. (We note that $\operatorname{col}_{C M}(A) \leq$ $\operatorname{col}_{C M}(A / x A)$ was proved ([7]) when a non zero-divisor $x \in \tau_{\omega}(A)$, where $\tau_{\omega}(A)$ is the trace of a canonical module $\omega$ in $A$.) 
Proposition 2.3. Let $(A, \mathfrak{m})$ be a Cohen-Macaulay local ring and $x$ be an $A$-regular element in $\mathrm{m}$. Then $\operatorname{row}_{C M}(A) \leq \operatorname{row}_{C M}(A / x A)$.

Proof. By Theorem 2.2, it is enough to show that

$$
\operatorname{row}_{C M}^{*}(A) \leq \operatorname{row}_{C M}^{*}(A / x A) \text {. }
$$

If $F_{0}: \cdots \rightarrow A^{n_{1}} \rightarrow A^{n_{0}} \rightarrow M \rightarrow 0$ is a minimal free resolution of a maximal Cohen-Macaulay $A$-module $M$, then $F_{\bullet} \otimes_{A} A / x A$ is also a minimal resolution of $M \otimes_{A} A / x A$ since $x$ is both $M$-regular and $A$-regular, i.e., $\operatorname{Tor}_{i}^{A}(A / x A, M)=0$ for all $i>0$. Note that $M \otimes_{A} A / x A$ is also a maximal Cohen-Macaulay $A / x A$ module ([7,Lemma 1.7]). Hence the conclusion follows from the definition of $\operatorname{row}_{C M}^{*}(A)$.

Corollary 2.4. Let $A$ be a Cohen-Macaulay local ring and $A \llbracket X \rrbracket$ a ring of formal power series over $A$. Then $\operatorname{row}_{C M}(A)=\operatorname{row}_{C M}(A \llbracket X \rrbracket)$.

Proof. Since the natural maps $A \rightarrow A \llbracket X \rrbracket$ and $A \llbracket X \rrbracket \rightarrow A \llbracket X \rrbracket / X A \llbracket X \rrbracket \cong$ $A$ are local homomorphisms of finite flat dimension, we have the inequalities $\operatorname{row}_{C M}(A) \leq \operatorname{row}_{C M}(A \llbracket X \rrbracket) \leq \operatorname{row}_{C M}(A \llbracket X \rrbracket / X A \llbracket X \rrbracket)=\operatorname{row}_{C M}(A)$, and so $\operatorname{row}_{C M}(A)=\operatorname{row}_{C M}(A \llbracket X \rrbracket)$.

We remark that the inequality in the above proposition may be strict. Let $(R, \mathfrak{m})$ be a regular local ring, and $x$ an $R$-regular element in $\mathfrak{m}^{2} \backslash \mathfrak{m}^{3}$. Then row $_{C M}(R)=1<2=\operatorname{row}_{C M}(R / x R$ ) by Theorem 2.5 (see Section 2 ) and Theorem $4.3([4])$.

To prove our main theorem, we use the technique of the mapping cone of complexes, which allows us to carry certain information on $\varphi_{i}(\operatorname{depth} M)$-steps to the left. We first recall the mapping cone ([2]): if $\alpha_{\bullet}:\left(F_{\bullet}, \varphi_{\bullet}\right) \rightarrow\left(G_{\bullet}, \psi_{\bullet}\right)$ is a map of complexes, then the mapping cone $\left(M\left(\alpha_{\bullet}\right)_{\bullet}, \Psi_{\bullet}\right)$ of $\alpha_{\bullet}$ is a complex such that

$$
M\left(\alpha_{\bullet}\right)_{i}=G_{i} \oplus F_{i-1} \quad \text { and } \quad \Psi_{i}=\left[\begin{array}{cc}
\psi_{i} & 0 \\
\alpha_{i-1} & -\varphi_{i-1}
\end{array}\right] .
$$

There is a long exact sequence of homologies arising from the exact sequence of complexes $0 \rightarrow G_{\bullet} \rightarrow M\left(\alpha_{\bullet}\right)_{\bullet} \rightarrow F_{\bullet}(-1) \rightarrow 0$ :

$$
\cdots \rightarrow \mathrm{H}_{i}\left(G_{\bullet}\right) \rightarrow \mathrm{H}_{i}\left(M\left(\alpha_{\bullet}\right)_{\bullet}\right) \rightarrow \mathrm{H}_{i-1}\left(F_{\bullet}\right) \rightarrow \mathrm{H}_{i-1}\left(G_{\bullet}\right) \rightarrow \cdots,
$$

where the connecting homomorphisms are induced by $\alpha_{\bullet}$.

It is known ([4, Lemma 2.1]) that if $\left(F_{\bullet}, \varphi_{\bullet}\right)$ is a minimal resolution of $M$,

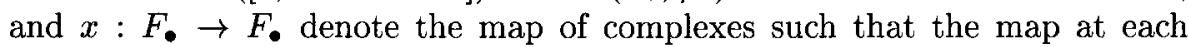
degree is a multiplication by $x \in \mathfrak{m}$, then $\left(M(x)_{\bullet}, \Psi_{\bullet}\right)$ is a minimal resolution of $M / x M$ for a non zero-divisor $x$ on $M$. The following is the main result of this paper.

Theorem 2.5. Let $(A, \mathfrak{m})$ be a Cohen-Macaulay local ring of dimension $d$. Then row $_{C M}(A)$ is finite, and moreover

$$
\operatorname{row}(A)=\operatorname{row}_{C M}(A) \text {. }
$$


Proof. By the definition of $\operatorname{row}(A)$, there is an $i>d$ such that the $i$-th map of a minimal free resolution of some module has a row which is contained in row $(A)-1$. Since $(i-1)$-th syzygy is a maximal Cohen-Macaulay module by the Ext characterization of depth and the long exact sequence of Ext, we have $\operatorname{row}(A) \leq \operatorname{row}_{C M}^{*}(A)=\operatorname{row}_{C M}(A)$ by Theorem 2.2 .

Suppose $\operatorname{row}_{C M}(A)=\infty$. Then for any $t>1$, there is a maximal CohenMacaulay module $M$ without free summands such that the minimal presenting matrix of $M$ has a row whose entries are contained in $\mathfrak{m}^{t-1}$. Using the same argument as below, we have $\operatorname{row}(A) \geq t$, which implies that $\operatorname{row}(A)$ is also infinite, which is a contradiction since row $(A)$ is always finite $([3$, Proposition $1.5]$ ). Hence $\operatorname{row}_{C M}(A)$ is finite.

For the other inequality, suppose $\operatorname{row}_{C M}(A)=t<\infty$. Then there exists a maximal Cohen-Macaulay module $M$ without free summands such that the minimal presenting matrix of $M$ has a row which is contained in $\mathfrak{m}^{t-1}$. Let $\left(F_{\bullet}, \varphi_{\bullet}\right)$ be a minimal resolution of $M$ :

$$
\left(F_{\bullet}, \varphi_{\bullet}\right): \cdots \longrightarrow A^{n_{i+1}} \stackrel{\varphi_{i+1}}{\longrightarrow} A^{n_{i}} \stackrel{\varphi_{i}}{\longrightarrow} A^{n_{i-1}} \longrightarrow \cdots \stackrel{\varphi_{1}}{\longrightarrow} A^{n_{0}} \longrightarrow 0 .
$$

Then we know that every entry of some row of $\varphi_{1}$ is contained in $\mathfrak{m}^{t-1}$. Choose a non zero-divisor $x_{1} \in \mathrm{m}^{t-1}$ of $M$. Then the mapping cone $\left(M\left(x_{1}\right), \Phi_{\bullet}\right)$ is a minimal resolution of $M / x_{1} M$ with

$$
\Phi_{q+1}=\left(\begin{array}{cc}
\varphi_{q+1} & 0 \\
x I & -\varphi_{q}
\end{array}\right) .
$$

Thus every entry of some row of $\Phi_{2}$ is contained in $\mathfrak{m}^{t-1}$. Since $M$ is a maximal Cohen-Macaulay module, we can construct a module $M /\left(x_{1}, \ldots, x_{d}\right) M$, by induction, for a $M$-regular sequence $x_{1}, \ldots, x_{d}$ in $\mathfrak{m}^{t-1}$ such that every entry of some row of $(d+1)$-th map of a minimal free resolution of $M /\left(x_{1}, \ldots, x_{d}\right) M$ is contained in $\mathfrak{m}^{t-1}$. Thus by definition of $\operatorname{row}(A)$, we know $t-1<\operatorname{row}(A)$; so $\operatorname{row}_{C M}(A) \leq \operatorname{row}(A)$. Hence $\operatorname{row}(A)=\operatorname{row}_{C M}(A)$.

\section{References}

[1] S. Ding, A note on the index of Cohen-Macaulay local rings, Comm. Algebra 21 (1993), no. $1,53-71$.

[2] D. Eisenbud, Commutative algebra, With a view toward algebraic geometry, Graduate Texts in Mathematics, 150. Springer-Verlag, New York, 1995.

[3] J. Koh and K. Lee, Some restrictions on the maps in minimal resolutions, J. Algebra 202 (1998), no. 2, 671-689.

[4] - New invariants of Noetherian local rings, J. Algebra 235 (2001), no. 2, 431-452.

[5] _ On column invariant and index of Cohen-Macaulay local rings, J. Korean Math. Soc. 43 (2006), no. 4, 871-883.

[6] K. Lee, Computation of numerical invariants col(-), row $(-)$ for a ring $k\left[\left[t^{e}, t^{e+1}\right.\right.$, $\left.t^{(e-1) e-1}\right]$, J. Korean Math. Soc. 37 (2000), no. 4, 521-530.

[7] - Column invariants over Cohen-Macaulay local rings, Comm. Algebra 33 (2005), no. 5, 1303-1314.

[8] G. J. Leuschke, Gorenstein modules, finite index, and finite Cohen-Macaulay type, Comm. Algebra 30 (2002), no. 4, 2023-2035. 
Department of Mathematics

SOOKMYUNG WOMEN'S UNIVERSITY

SEOUL 140-742, KoREA

E-mail address: kilee@sookmyung.ac.kr 